\title{
Darf Pflegepersonal bald den Arzt ersetzen?
}

- Das Bundesministerium für Gesundheit (BMG) hat die Richtlinie zur Übertragung ärztlicher Tätigkeiten auf Alten- und Krankenpfleger im Rahmen von Modellprojekten nicht beanstandet. Damit kann die Heilkundeübertragungs-Richtlinie in Kraft treten, sobald sie im Bundesanzeiger veröffentlicht wurde.

Der Gemeinsame Bundesausschuss hatte die Richtlinie am 20. Oktober 2011 beschlossen. Sie regelt die Übertragung von ärztlichen Tätigkeiten auf Alten- und Krankenpflegepersonal zur selbständigen Ausübung von Heilkunde im Rahmen von Modellvorhaben nach $\S 63$ Absatz 3c SGB V.

\section{MMW Kommentar}

Teil A der Richtlinie enthält verbindliche Regelungsinhalte sowie Empfehlungen zu den Inhalten der Modellvorhaben: Im Rahmen dieser Modelle können ärztliche Tätigkeiten zukünftig auf Berufsangehörige der Alten- und Krankenpflege zur selbstständigen und eigenverantwortlichen Ausübung übertragen werden. Dazu zählen sowohl prozedurenspezifische Leistungen (wie Blutentnahme) als auch diagnosebezogene Versorgungskonzepte (beispielsweise bei Diabetes mellitus).

Diagnose- und Indikationsstellung bleiben weiterhin in ärztlicher Hand, daran sind die Kranken- und Altenpfleger gebunden. Sie dürfen Überweisungen durch Vertragsärzte veranlassen, nicht aber selbst durchführen. Alten- und Krankenpflegekräfte dürfen im Rahmen der Modellvorhaben bestimmte, noch zu definierende, Medizinprodukte, Hilfsmittel sowie in der Richtlinie abschlieBend aufgeführte Heilmittel selbst verordnen. Darüber hinaus werden in der Richtlinie weitere Details der Modellvorhaben geregelt, etwa sachliche, personelle und organisatorische Voraussetzungen.
Teil B der Richtlinie benennt die durchführbaren Tätigkeiten (in Form von Katalogen) sowie Qualitätsanforderungen für einzelne Tätigkeiten. Ein diagnosebezogener Katalog umfasst folgende Krankheitsbilder: Diabetes mellitus Typ 1 und 2, chronische Wunden, Demenz (nicht palliativ), Hypertonus (ohne Schwangerschaft). Ein zweiter Katalog enthält prozedurenbezogene Einzeltätigkeiten. Dazu gehören zum Beispiel: Infusionstherapie/Injektionen, Anlage und Versorgung einer Magensonde, Ableitungen, Entlastungen, Zugänge und Patientenmanagement.

Die Alten- und Krankenpflegekräfte müssen ihre erworbene Qualifikation in einer staatlichen Prüfung nachweisen. In den jeweiligen Berufsgesetzen sind Regelungen zu den Ausbildungen festzulegen, die die berufsrechtlichen Voraussetzungen für die Übertragung ärztlicher Tätigkeiten schaffen. Diesen Regelungen kann nicht durch verbindliche Qualifikationsanforderungen vorgegriffen werden, die in der Richtlinie formuliert sind. Die hausärztlichen Praxen in der Fläche sind durch diese Regelungen zunächst nicht betroffen, denn es handelt sich um eine auf höchstens fünf Jahre begrenzte Erprobung der Übertragung von Heilkunde im Rahmen von Modellvorhaben, an denen Ärzte, Krankenkassen und die Pflege beteiligt sein müssen. Nach abschließender Evaluation wird darüber zu beraten sein, ob und unter welchen Voraussetzungen diese Heilkundeübertragung nach \$63 Absatz 3c SGBV flächendeckend umgesetzt wird.

\section{Achtung:}

\section{Hier muss der Dummy durch eine Anzeige ersetzt werden!!}

